OPEN ACCESS

Edited by:

Pei-Ning Wang,

National Yang-Ming University, Taiwan

Reviewed by:

Pasquale Mone,

Second University of Naples

(SUN), Italy

Allan Bregola,

University Hospitals Bristol NHS

Foundation Trust, United Kingdom

Tian-Long Wang,

Capital Medical University, China

*Correspondence:

Jialing Wu

wywil2009@hotmail.com

Specialty section:

This article was submitted to Dementia and Neurodegenerative

Diseases,

a section of the journal

Frontiers in Neurology

Received: 08 October 2021 Accepted: 25 November 2021

Published: 06 January 2022

Citation:

Mu L, Jiang L, Chen J, Xiao M, Wang W, Liu P and Wu J (2022) Serum Inflammatory Factors and

Oxidative Stress Factors Are Associated With Increased Risk of Frailty and Cognitive Frailty in Patients With Cerebral Small Vessel Disease. Front. Neurol. 12:786277. doi: 10.3389/fneur.2021.786277

\section{Serum Inflammatory Factors and Oxidative Stress Factors Are Associated With Increased Risk of Frailty and Cognitive Frailty in Patients With Cerebral Small Vessel Disease}

\author{
Lei Mu ${ }^{1,2}$, Limin Jiang ${ }^{2}$, Juan Chen ${ }^{2}$, Mei Xiao ${ }^{2}$, Wei Wang ${ }^{3}$, Peipei Liu ${ }^{3}$ and Jialing Wu ${ }^{3 *}$ \\ ${ }^{1}$ Clinical College of Neurology, Neurosurgery and Neurorehabilitation, Tianjin Medical University, Tianjin, China, ${ }^{2}$ Department \\ of Geriatrics, Inner Mongolia People's Hospital, Hohhot, China, ${ }^{3}$ Department of Neurorehabilitation and Neurology, Tianjin \\ Huanhu Hospital, Tianjin Key Laboratory of Cerebral Vascular and Neurodegenerative Diseases, Tianjin Neurosurgical \\ Institute, Tianjin, China
}

Objective: To study the correlation between serum inflammatory factors, oxidative stress factors and frailty, and cognitive frailty in patients with cerebral small vessel disease (CSVD).

Methods: A total of 281 patients with CSVD were selected from Tianjin Huanhu Hospital and Inner Mongolia People's Hospital from March 2019 to March 2021. CSVD was diagnosed by MRI. The FRAIL scale was used to evaluate the frailty of patients. Patients with CSVD with frailty and MMSE score $<27$ were considered to have cognitive frailty. Patients with non-cognitive frailty were included in the control group. The Montreal Cognitive Assessment (MoCA) and Mini-Mental State Examination (MMSE) were used to evaluate the cognitive function of patients with CSVD. The serum interleukin 6 (IL-6), tumor necrosis factor-alpha (TNF- $\alpha$ ), matrix metalloproteinase 3 (MMP-3), superoxide dismutase (SOD), and malondialdehyde (MDA) of patients with CSVD were detected. The correlation between blood inflammatory factors and oxidative stress factors with the frailty and cognitive frailty patients of CSVD were analyzed. Univariate and multivariate logistic regression were used to analyze the correlation between cognitive frailty and CSVD.

Results: Among the patients with CSVD selected in this study, female patients and older patients had a higher proportion of frailty $(p<0.001)$. In the Frail group, MoCA score and MMSE score were significantly lower than in the Pre-Frail and Robust groups, Hamilton Depression Scale (HAMD) and Hamilton Anxiety Scale (HAMA) scores were significantly higher than the Pre-Frail and Robust groups, and the differences were statistically significant ( $p<0.05$ ). Serum CRP, IL-6, TNF- $\alpha$, MMP-3, and MDA levels in the Frail group were higher, but SOD levels were lower. The levels of serum CRP, IL-6, TNF- $\alpha$, MMP-3, and MDA in patients with CSVD in the Cognitive Frailty group were significantly higher than those of the Control group, while the levels of SOD were significantly lower than those of the Control group, and the differences were significant $(p<0.001)$. The results of univariate and multivariate logistic regression analysis showed that CRP, 
TNF- $\alpha$, MMP-3, and MDA levels were associated with cognitive frailty in patients with CSVD $(p<0.05)$.

Conclusion: The increase of serum CRP, TNF- $\alpha$, MMP-3, and MDA levels are significantly related to the increased risk of frailty and cognitive frailty in patients with CSVD.

Keywords: cerebral small vascular disease, cognitive frailty, frailty, CRP, TNF- $\alpha$, MMP-3, MDA

\section{INTRODUCTION}

Cerebral small vessel disease (CSVD) is a common clinical cerebrovascular disease with a high incidence. Small perforating arterioles, capillaries, and venules of the brain are the most common causes of vascular cognitive disorders and dementia (1-3). With the acceleration of population aging and the high incidence of risk factors for cerebrovascular diseases, CSVD is increasing, which not only increases the social burden but also reduces the quality of life of patients. Therefore, it is of great significance to study the pathogenesis of CSVD and improve the clinical diagnosis and treatment of patients with CSVD.

Frailty is a physiological decline syndrome occurring with aging, which is characterized by reduced functional reserve and increased vulnerability (4). Frail people are less able to adapt to stress factors such as disease or trauma (5). Frailty is closely related to disability, fall, hospitalization, readmission, and death (6). In the process of human aging, all people will have cognitive decline, and some elderly people will eventually suffer cognitive impairment and dementia. Cognitive frailty refers to a clinical syndrome of reduced cognitive reserve (except dementia) combined with physical weakness (7). The specific mechanism of frailty is currently unclear.

In recent years, inflammatory factors have been recognized as risk factors for stroke (8), dementia $(9,10)$, small vessel disease $(11,12)$, and frailty (13). Research evidence showed that there was a significant correlation between CSVD and vascular inflammatory factors $(14,15)$.

The oxidative stress pathway plays an important role in the frailty and cognitive impairment of patients with CSVD. Oxidative stress can cause lipid oxidative damage, while malondialdehyde (MDA) is a common end product of lipid peroxidation, and increased MDA levels are generally considered to be a sign of oxidative stress pathway activation. Therefore, MDA can indirectly reflect the metabolism of oxygen free radicals in the body $(16,17)$. Superoxide dismutase (SOD) is an important antioxidant enzyme with strong oxidizing properties in the human body, which can effectively eliminate excess oxygen free radicals and derivatives of oxygen free radicals, and protect cells from damage (18). The specific mechanism of oxidative stress in the frailty and cognitive dysfunction of patients with CSVD is still unclear.

Therefore, inflammatory factors and oxidative stress factors may be one of the possible mechanisms of frailty and cognitive frailty. However, the pathogenesis of frailty and cognitive frailty in patients with CSVD is still unclear. This study aimed to study the effects of inflammation and oxidative stress on frailty and cognitive frailty in patients with CSVD and to provide evidence for intervention frailty and cognitive frailty.

\section{METHODS}

\section{Patients}

A total of 281 patients with CSVD (including lacunar infarction, high white matter signal, microhemorrhage, perivascular space, and brain atrophy) diagnosed by head MRI in Tianjin Huanhu Hospital and Inner Mongolia People's Hospital from March 2019 to March 2021 were collected, aged 37-90 years old, with an average of $65.95 \pm 9.07$ years old. The inclusion criteria were (1) stable health status, able to move independently, able to understand and correctly answer questions, and assist in completing questionnaires; and (2) informed consent and voluntary participation in the study. The exclusion criteria were (1) patients with severe physical dysfunction, unable to cooperate with the completion of the evaluation, and accompanying visitors; (2) people with speech or hearing impairment; (3) severe liver and kidney diseases, vital organ failure, and expected survival of $<1$ year; (4) acute infection; (5) those with contraindications to magnetic resonance examination; (6) cognitive frailty caused by factors such as Alzheimer's disease, Parkinson's disease, sequelae of cerebral infarction, and severe demyelinating disease; (7) those who have taken nootropic drugs for a long time in the past; and (8) those who have communication difficulties and are unable to complete the neuropsychological assessment.

\section{FRAIL Scale}

The FRAIL scale consists of five self-reported components. (1) Fatigue: in the past 4 weeks, have you often felt tired? (2) Resistance: if you do not have a rest or do not have mobility aids to climb 10 stairs, do you find it difficult? (3) Ambulation: do you find it difficult to walk 500 600 m without the assistance of mobility aids? (4) Illnesses: do you have five or more of the following diseases: hypertension, diabetes, acute heart attack, stroke, malignant tumors, congestive heart failure, asthma, arthritis, chronic lung disease, kidney disease, and angina pectoris? (5) Loss of weight: in the past 1 year or less, has your body weight decreased by $\geq 5 \%$ ? (19). Patients having three or more of the above can be diagnosed as Frail, meeting one or two of the above can be diagnosed as Pre-Frail, and those who do not meet any of the above are diagnosed as Robust $(20,21)$. 


\section{Assessment of Cognitive Function}

The Montreal Cognitive Assessment Scale (MoCA) (22) and Mini-mental State Examination (MMSE) were used to assess the cognitive function of patients with CSVD (23).

The Montreal Cognitive Assessment Scale includes 10 subtests, including visuospatial/executive abilities, naming, attention to digits, attention to letters, attention to subtraction, language repetition, language fluency, abstraction, delayed recall, and orientation. The total score is 30 points. The MoCA score needs to refer to the educated years of the evaluator. If the educated period of the evaluator is $\leq 12$ years, one point should be added to the measured score to correct the bias caused by the level of education.

The total score of MMSE is 30. A score of 27-30 indicates normal cognitive function, a score of $<27$ indicates cognitive dysfunction.

In this study, patients with CSVD with frailty and an MMSE score $<27$ were considered to have cognitive frailty (23).

\section{Emotional Assessment}

The Hamilton Depression Scale (HAMD) (24) and Hamilton Anxiety Scale (HAMA) (25) were used to evaluate the mood of the patient.

\section{Laboratory Index Testing}

All patients fasted for $12 \mathrm{~h}$ in the morning and then $10 \mathrm{ml}$ of peripheral venous blood was drawn. Serum inflammatory factors and oxidative stress factors, such as C-reactive protein (CRP), interleukin-6 (IL-6), tumor necrosis factor $\alpha$ (TNF- $\alpha$ ), matrix metalloproteinase-3 (MMP-3), superoxide dismutase (SOD), and malondialdehyde (MDA) were detected by using ELISA.

\section{Statistical Analysis}

The data were analyzed by SPSS (version 22.0, Chicago, IL, USA), measurement data were presented as mean $\pm \mathrm{SD}$, ANOVA, $t$ test was used for statistical analysis between groups, and $\chi^{2}$-test was used for categorical variables $[n(\%)]$. The frailty was used as the dependent variable, and the clinical data and the level of inflammatory factors and oxidative stress factors were used as independent variables to conduct a univariate regression analysis. Cognitively frailty was used as the dependent variable, and the statistically different indicators of univariate regression analysis were used as independent variables to perform multivariate logistic regression analysis. Univariate and multivariate logistic regressions were used to analyze the association between cognitive frailty with CSVD. All test results were two-tailed, and $p<0.05$ indicated that the difference was statistically significant.

\section{RESULTS}

\section{FRAIL and Demographic Data}

The correlation between FRAIL score and gender, age, BMI, hypertension, smoking, and drinking is shown in Table 1. The analysis results showed that among the patients with CSVD selected in this study, female patients and older patients had a higher proportion of frailty $(p<0.001)$. There was no significant
TABLE 1 | Comparison of demographic information between the Frail, Pre-Frail, and Robust groups.

\begin{tabular}{lcccc}
\hline & $\begin{array}{c}\text { Frail } \\
(\boldsymbol{n}=\mathbf{3 2})\end{array}$ & $\begin{array}{c}\text { Pre-Frail } \\
(\boldsymbol{n}=\mathbf{1 1 2})\end{array}$ & $\begin{array}{c}\text { Robust } \\
(\boldsymbol{n}=\mathbf{1 3 7})\end{array}$ & $\boldsymbol{p}$ \\
\hline Gender [n (\%)] & & & & $<0.001$ \\
$\quad$ Male & $11(34.38 \%)$ & $78(69.64 \%)$ & $109(79.56 \%)$ & \\
Female & $21(65.63 \%)$ & $34(30.36 \%)$ & $28(20.44 \%)$ & \\
Age (years) & & & & \\
BMl (kg/m $)^{2}$ & $74.78 \pm 9.62$ & $67.91 \pm 7.47$ & $62.28 \pm 8.23$ & $<0.001$ \\
Hypertension $[n(\%)]$ & $24.59 \pm 3.35$ & $24.71 \pm 2.96$ & $25.73 \pm 2.75$ & 0.38 \\
Yes & $27(84.38 \%)$ & $99(88.39 \%)$ & $113(82.48 \%)$ & 0.43 \\
No & $5(15.63 \%)$ & $13(11.61 \%)$ & $24(17.52 \%)$ & \\
Smoking [n (\%)] & & & & 0.41 \\
Yes & $11(34.38 \%)$ & $45(40.18 \%)$ & $63(45.99 \%)$ & \\
No & $21(65.63 \%)$ & $67(59.82 \%)$ & $74(54.01 \%)$ & \\
Drinking [n (\%)] & & & & 0.07 \\
Yes & $4(12.50 \%)$ & $31(27.68 \%)$ & $45(32.85 \%)$ & \\
No & $28(87.50 \%)$ & $81(72.32 \%)$ & $92(67.15 \%)$ & \\
\hline
\end{tabular}

$B M I$, body mass index. ${ }^{a}$ Data are expressed as mean $\pm S D$.

TABLE 2 | Comparison of Montreal Cognitive Assessment Scale (MoCA), Mini-mental State Examination (MMSE), Hamilton Depression Scale (HAMD), and Hamilton Anxiety Scale (HAMA) scores among the Frail, Pre-Frail, and Robust groups.

\begin{tabular}{lcccc}
\hline & $\begin{array}{c}\text { Frail } \\
(\boldsymbol{n}=\mathbf{3 2})\end{array}$ & $\begin{array}{c}\text { Pre-Frail } \\
(\boldsymbol{n}=\mathbf{1 1 2})\end{array}$ & $\begin{array}{c}\text { Robust } \\
(\boldsymbol{n}=\mathbf{1 3 7})\end{array}$ & $\boldsymbol{p}$ \\
\hline MoCA $^{\mathrm{a}}$ & $18.16 \pm 4.00$ & $20.32 \pm 4.03$ & $22.96 \pm 3.62$ & $<0.001$ \\
MMSE $^{\mathrm{a}}$ & $22.84 \pm 3.71$ & $24.82 \pm 3.07$ & $26.55 \pm 2.35$ & $<0.001$ \\
HAMD $^{\mathrm{a}}$ & $6.16 \pm 3.14$ & $3.72 \pm 2.72$ & $2.39 \pm 2.12$ & $<0.001$ \\
HAMA $^{\mathrm{a}}$ & $6.38 \pm 2.84$ & $3.79 \pm 2.46$ & $2.58 \pm 1.83$ & $<0.001$
\end{tabular}

${ }^{a}$ Data are expressed as mean $\pm S D$.

correlation between BMI, hypertension, smoking, drinking, and frailty $(p>0.05)$.

\section{Correlation Between Frailty and MoCA, MMSE, HAMD, and HAMA Scales}

The correlation between frailty and MoCA and MMSE scores is shown in Table 2. In the Frail group, MoCA and MMSE scores were significantly lower than the Pre-Frail and Robust groups, HAMD and HAMA scores were significantly higher than the PreFrail and Robust groups, and the differences were statistically significant $(p<0.05)$.

\section{Frailty Was Correlated With Levels of Inflammatory Factors and Oxidative Stress Factors}

The comparison results of the inflammatory factors and oxidative stress factors between the Frail, Pre-Frail, and Robust groups are shown in Figure 1. The CRP, IL-6, TNF- $\alpha$, MMP-3, SOD, and MDA levels of patients with CSVD between Frail, Pre-Frail, and 


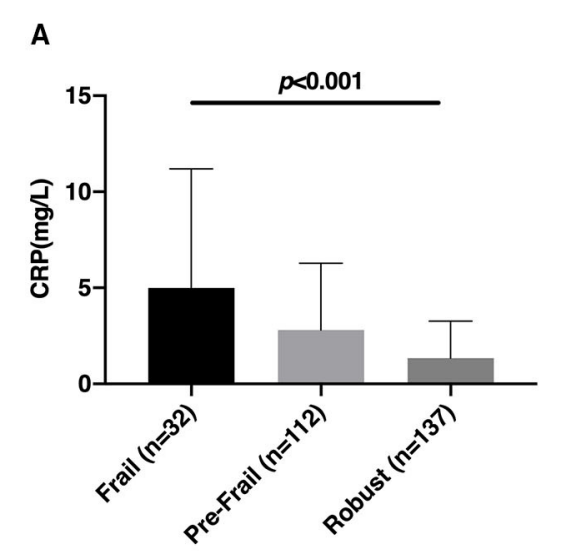

B

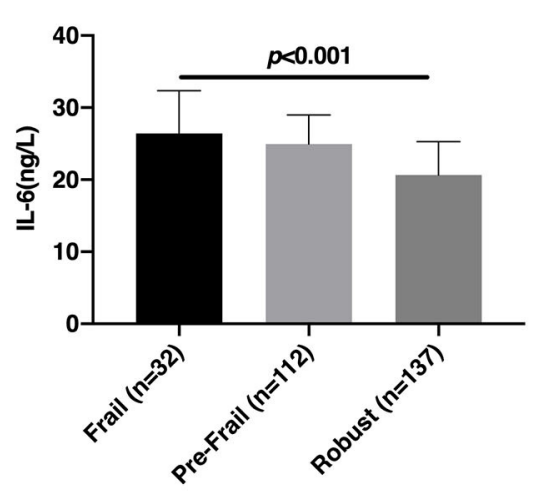

E

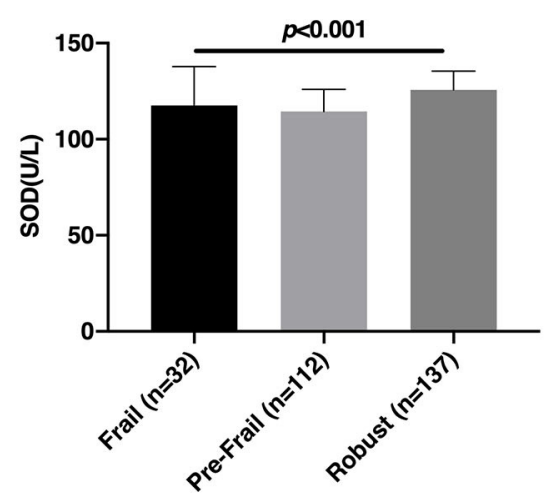

C

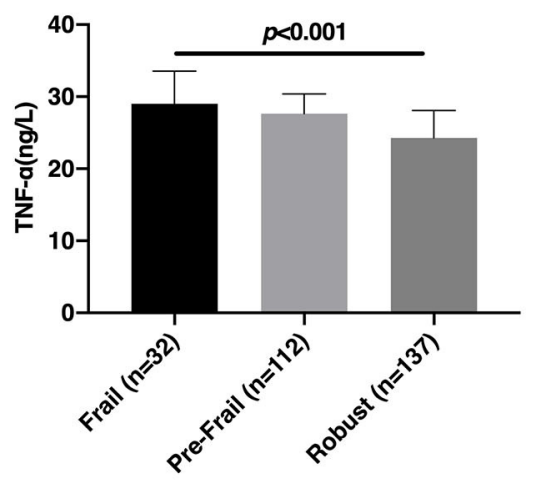

$\mathbf{F}$

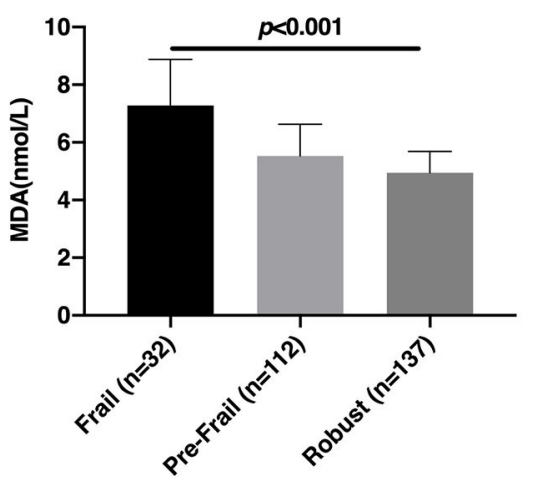

FIGURE 1 | Comparison of the levels of inflammatory factors and oxidative stress factors among the Frail, Pre-Frail, and Robust groups. The comparison of serum CRP (A), IL-6 (B), TNF- $\alpha$ (C), MMP-3 (D), SOD (E), and MDA (F) levels between the Frail, Pre-Frail, and Robust groups. CRP, C-reactive protein; IL-6, Interleukin-6; TNF- $\alpha$, tumor necrosis factor $\alpha$; MMP-3, matrix metalloproteinase-3; SOD, superoxide dismutase; MDA, malondialdehyde.

Robust groups are significantly different $(p<0.05)$. Serum CRP, IL-6, TNF- $\alpha$, MMP-3, and MDA levels in the Frail group were higher, but SOD levels were lower.

\section{Cognitive Frailty and Demographic Data of Patients With CSVD}

The demographic data of patients with CSVD in the Cognitive Frailty and Non-Cognitive Frailty (Control) groups are shown in Table 3. In the Cognitive Frailty group, the number of women in patients with CSVD and their age were significantly higher than those in the Control group, and the difference was statistically significant $(p<0.001)$, there was no statistically significant difference between the BMI, hypertension, smoking, and drinking for patients with CSVD in the Cognitive Frailty and Control groups $(p>0.05)$.

\section{Correlation Between Cognitive Frailty and HAMD and HAMA Scores in Patients With CSVD}

The correlation between cognitive frailty and HAMD and HAMA scores in the patients with CSVD is shown in Table 4. In the Cognitive Frailty group, the HAMD and HAMA scores of patients with CSVD were significantly higher than those of the Control group, and the differences were statistically significant $(p<0.001)$.

\section{Correlation Between Cognitive Frailty and the Levels of Inflammatory Factors and Oxidative Stress Factors in Patients With CSVD}

The correlation between cognitive frailty and CRP, IL-6, TNF$\alpha$, MMP-3, SOD, and MDA in patients with CSVD is shown in Figure 2. The levels of serum CRP, IL-6, TNF- $\alpha$, MMP-3, and MDA in patients with CSVD with cognitive frailty were significantly higher than those of the Control group, while the level of SOD was significantly lower than those of the Control group, and the differences were extremely significant $(p<0.001)$.

\section{Univariate Logistic Regression Analysis}

We performed a univariate logistic regression analysis of the subjects' demographic data, serum inflammatory factors, and oxidative stress factors, and the results are shown in Table 5. 
TABLE 3 | Comparison of demographic data of patients with cerebral small vessel disease (CSVD) in the Cognitive Frailty and Control groups.

\begin{tabular}{lccc}
\hline & $\begin{array}{c}\text { Cognitive frailty } \\
(\boldsymbol{n}=\mathbf{2 6})\end{array}$ & $\begin{array}{c}\text { Control } \\
(\boldsymbol{n}=\mathbf{2 5 5})\end{array}$ & $\boldsymbol{p}$ \\
\hline Gender $[n(\%)]$ & & & $<0.001$ \\
$\quad$ Male & $9(34.62 \%)$ & $189(74.12 \%)$ & \\
$\quad$ Female & $17(65.38 \%)$ & $66(25.88 \%)$ & \\
Age (years) & $76.00 \pm 9.64$ & $64.93 \pm 8.38$ & $<0.001$ \\
BMl (kg/m²) & $24.48 \pm 2.99$ & $25.27 \pm 2.93$ & 0.19 \\
Hypertension $[n(\%)]$ & & & 0.52 \\
$\quad$ Yes & $21(80.77 \%)$ & $218(85.49 \%)$ & \\
$\quad$ No & $5(19.23 \%)$ & $37(14.51 \%)$ & \\
Smoking $[n(\%)]$ & & & 0.40 \\
Yes & $9(34.62 \%)$ & $110(43.14 \%)$ & \\
$\quad$ No & $17(65.38 \%)$ & $145(56.86 \%)$ & \\
Drinking $[n(\%)]$ & & & 0.12 \\
Yes & $4(15.38 \%)$ & $76(29.80 \%)$ & \\
No & $22(84.62 \%)$ & $179(70.20 \%)$ & \\
\hline
\end{tabular}

$B M I$, body mass index. ${ }^{a}$ Data are expressed as mean $\pm S D$.

TABLE 4 | Comparison of HAMD and HAMA scores between Cognitive Frailty and Control groups.

\begin{tabular}{lccc}
\hline & Cognitive frailty $(\boldsymbol{n}=\mathbf{2 6})$ & Control $(\boldsymbol{n}=\mathbf{2 5 5})$ & $\boldsymbol{p}$ \\
\hline HAMD $^{\mathrm{a}}$ & $6.04 \pm 3.32$ & $3.07 \pm 2.55$ & $<0.001$ \\
HAMA $^{\mathrm{a}}$ & $6.31 \pm 3.08$ & $3.21 \pm 2.26$ & $<0.001$
\end{tabular}

HAMD, Hamilton Depression Scale; HAMA, Hamilton anxiety scale. ${ }^{a}$ Data are expressed as mean $\pm S D$.

Univariate analysis showed that gender, age, BMI, CRP, IL-6, TNF- $\alpha$, MMP-3, and MDA were all associated with cognitive frailty in patients with CSVD $(p<0.05)$. There was no significant correlation between hypertension, smoking, drinking, SOD, and the risk of cognitive frailty in patients with CSVD $(p>0.05)$.

\section{Multivariate Logistic Regression Analysis}

We used multivariate logistic regression to analyze the correlation between cognitive frailty and CSVD, and adjusted the demographic data of the patients with CSVD. The analysis results are shown in Table 6. The results showed that CRP, TNF- $\alpha$, MMP-3, and MDA levels were associated with cognitive frailty in patients with CSVD $(p<0.05)$.

\section{DISCUSSION}

According to the results of this study, women and older people were at greater risk of frailty and cognitive frailty, which was consistent with the findings of Kang et al. (26). Kim et al. (27) found that age and gender were important factors affecting frailty. Women were more likely to have frailty symptoms than men, which may be related to female physiological characteristics. Elderly patients are more prone to frailty symptoms due to decreased physical function and more complicated diseases.
Solfrizzi et al. (28) found that with the increase of age, the incidence of cognitive frailty in elderly women was higher than that in elderly men. According to our research results, frailty and cognitive frailty patients were more likely to have emotional disorders, these symptoms may be a challenge to the patient's normal life. Therefore, it can be considered that improving the frailty and cognitive frailty symptoms of patients with CSVD has important clinical significance.

The results of this study showed that compared with the PreFrail and Robust groups, the serum CRP, IL-6, TNF- $\alpha$, MMP3 , and MDA levels of patients with CSVD in the Frail group were higher, and the SOD level was lower. The study found that in patients with mild cognitive frailty and mild to moderate Alzheimer's disease, the pro-inflammatory factor TNF- $\alpha$ was associated with an increased risk of physical frailty (29). Similarly, studies have shown that TNF- $\alpha$ is associated with the frailty phenotype (30). In a longitudinal study, IL-6 was found to be associated with frailty (31). Another study found that IL-6 levels increased with age and were negatively correlated with exercise endurance in frail elderly people (32). With age, the levels of serum IL-6, CRP, and TNF-a increase significantly, which were positively correlated with the decline of muscle mass, strength, and function, and promoted the occurrence of frailty (33). We know that inflammatory factors, such as IL-6, CRP, and TNF$\alpha$ can directly or indirectly affect motor function, endocrine function, circulatory function, and neurological function of patients with CSVD and further participate in the occurrence, progression, and outcome of frailty. Ingles et al. (34) found that MAD, a product of plasma lipid peroxidation, was increased in the Frailty group of elderly people in the community, which proved to be related to frailty.

After using multivariate logistic regression analysis and adjusting the demographic data, we found that CRP, TNF$\alpha$, MMP-3, and MDA were associated with cognitive frailty in patients with CSVD. Studies have found that the use of dexmedetomidine can reduce IL-6 levels, improve long-term cognitive dysfunction and anti-inflammatory effects (35). In addition, in a rat model, researchers found that dexmedetomidine can reduce the expression levels of TNF- $\alpha$ and NF- $\kappa$ B and improve postoperative cognitive function in rats (36). Another study showed that MMP-3 may be involved in the early pathogenesis of Alzheimer's disease, which may lead to neuronal degeneration, neurofibrillary tangles, and cognitive dysfunction (37). MDA is an independent risk factor for postoperative cognitive dysfunction in elderly patients undergoing hip fracture surgery (38). The results of a study showed that luteolin can prevent the increase of TNF- $\alpha$, IL- $1 \beta$, and IL- 6 , and MDA, increase the activity of SOD and glutathione peroxidase, inhibit the excessive activation of microglia and the proliferation of astrocytes (especially in the hippocampus and cortex), and improve learning and learning ability, which is expected to improve the cognitive dysfunction of patients (39). The above evidence shows that CRP, TNF- $\alpha, \mathrm{MMP}-3$, and MDA are expected to become targets for improving the cognitive function of patients with CSVD, and have potential clinical therapeutic value. Although IL-6 and SOD levels were not found in this study to be associated with the risk of cognitive frailty in patients 

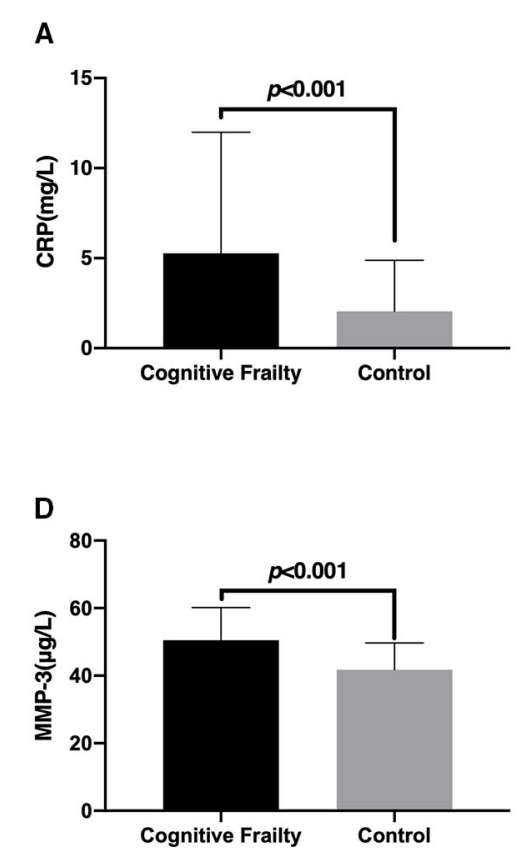

B
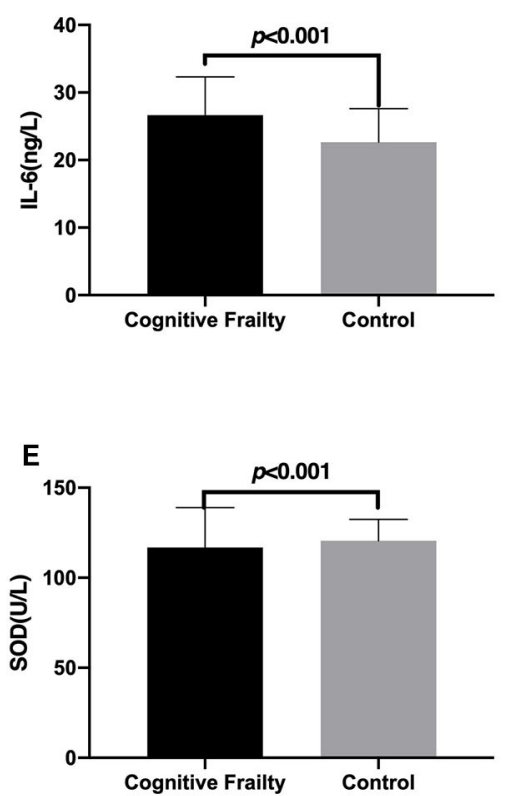

C

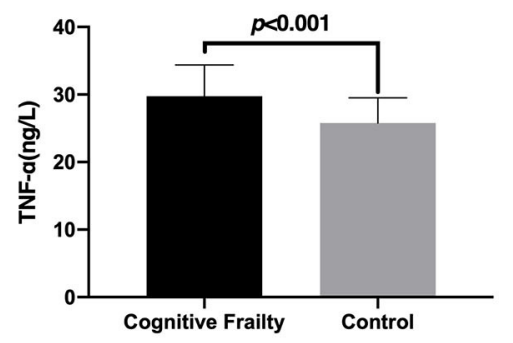

F

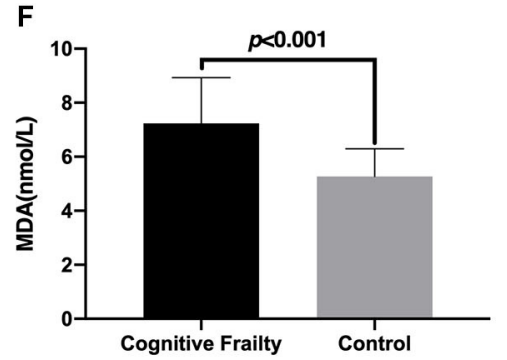

FIGURE 2 | Comparison of the levels of inflammatory factors and oxidative stress factors in patients with cerebral small vessel disease (CSVD) between the Cognitive Frailty and Control groups. The comparison of serum CRP (A), IL-6 (B), TNF- $\alpha$ (C), MMP-3 (D), SOD (E), and MDA (F) levels of patients with CSVD between the Cognitive Frailty and Control groups. CRP, C-reactive protein; IL-6, Interleukin-6; TNF- $\alpha$, tumor necrosis factor $\alpha$; MMP-3, matrix metalloproteinase-3; SOD, superoxide dismutase; MDA, malondialdehyde.

with CSVD, we speculate that the reason may be related to the small sample size of patients with CSVD with cognitive frailty, therefore, we plan to further expand the sample size for research in the later period.

Studies have found that inflammation in the brain can promote the release of peripheral blood inflammatory factors, and these factors can change the permeability of the bloodbrain barrier, thereby promoting the entry of inflammatory factors into the brain $(40,41)$. After inflammatory factors enter the brain, they can induce brain-related diseases. For example, inflammatory factors play a key role in the occurrence of Alzheimer's disease (AD), which has been supported by research evidence $(42,43)$. In addition, the latest research evidence showed that patients who had recovered from COVID-19 had cognitive dysfunction, which may be related to the concentration of CRP inflammatory factors (44). Researchers have also found that intravenous injection of dexmedetomidine can reduce serum inflammatory factors and improve the cognitive function of patients undergoing tonsillectomy (45).

In addition, this study found that the MoCA score and MMSE score of the Cognitive Frailty group were significantly lower than those of the Control group, suggesting that cognitive frailty in patients with CSVD is closely related to the occurrence of cognitive dysfunction, but its internal linkage mechanism needs further study.

We know that the causes of frailty and cognitive frailty are very complex $(46,47)$. Taken together, they involve the interaction of genetic, physiological, psychological, social, and
TABLE 5 | Univariate logistic regression analysis results of cognitive frailty in patients with CSVD.

\begin{tabular}{lccccccc}
\hline & B & SE & Wald & Sig. & Exp(B) & \multicolumn{2}{c}{$\mathbf{9 5 \%}$ CI } \\
\cline { 6 - 8 } & & & & & & Lower & Upper \\
\hline Female & 1.69 & 0.44 & 14.97 & $<0.01$ & 5.41 & 2.30 & 12.72 \\
Age & -2.17 & 1.03 & 4.43 & 0.04 & 0.12 & 0.02 & 0.86 \\
BMI & 1.18 & 0.43 & 7.74 & 0.01 & 3.26 & 1.42 & 7.49 \\
Hypertension & 0.34 & 0.53 & 0.41 & 0.52 & 1.40 & 0.50 & 3.95 \\
Smoking & 0.36 & 0.43 & 0.70 & 0.40 & 1.43 & 0.62 & 3.34 \\
Drinking & 0.85 & 0.56 & 2.29 & 0.13 & 2.34 & 0.78 & 7.01 \\
CRP & 0.15 & 0.04 & 13.53 & $<0.01$ & 1.16 & 1.07 & 1.26 \\
IL-6 & 0.15 & 0.04 & 13.22 & $<0.01$ & 1.16 & 1.07 & 1.26 \\
TNF- $\alpha$ & 0.27 & 0.06 & 21.09 & $<0.01$ & 1.31 & 1.17 & 1.47 \\
MMP-3 & 0.12 & 0.03 & 21.53 & $<0.01$ & 1.13 & 1.07 & 1.19 \\
SOD & -0.01 & 0.02 & 0.06 & 0.81 & 1.00 & 0.96 & 1.04 \\
MDA & 0.99 & 0.16 & 37.16 & $<0.01$ & 2.68 & 1.95 & 3.69 \\
\hline
\end{tabular}

The adjusted $R^{2}$-values of regression analysis for gender, age, BMI, hypertension, smoking, drinking, CRP, IL-6, TNF- $\alpha$, MMP-3, SOD, and MDA were 0.060, 0.019, $0.026,0.001,0.002,0.005,0.068,0.048,0.081,0.086,0.004$, and 0.211, respectively. $B M I$, body mass index; CRP, C-reactive protein; IL-6, Interleukin-6; TNF- $\alpha$, tumor necrosis factor $\alpha$; MMP-3, matrix metalloproteinase-3; $S O D$, superoxide dismutase; MDA, malondialdehyde; SE, standard error; $\mathrm{Cl}$, confidence interval.

environmental factors (48). A number of studies have shown the relationship between inflammation, oxidative stress, frailty, and cognitive frailty (49-51). These studies suggest that we 
TABLE 6 | Multivariate logistic regression analysis results of cognitive frailty in patients with CSVD.

\begin{tabular}{lccccccc}
\hline & B & SE & Wald & Sig. & Exp(B) & \multicolumn{2}{c}{$95 \%$ Cl } \\
\cline { 6 - 8 } & & & & & & Lower & Upper \\
\hline CRP & 0.99 & 0.45 & 4.82 & 0.03 & 2.69 & 1.11 & 6.49 \\
IL-6 & 0.81 & 0.52 & 2.49 & 0.12 & 2.26 & 0.82 & 6.21 \\
TNF- $\alpha$ & 1.57 & 0.59 & 7.14 & 0.01 & 4.81 & 1.52 & 15.23 \\
MMP-3 & 1.52 & 0.58 & 6.85 & 0.01 & 4.56 & 1.46 & 14.19 \\
SOD & -0.38 & 0.45 & 0.71 & 0.40 & 0.69 & 0.29 & 1.65 \\
MDA & 2.15 & 0.65 & 10.87 & 0.00 & 8.61 & 2.39 & 30.95 \\
\hline
\end{tabular}

$R^{2}=0.452$. BMI, body mass index; CRP, C-reactive protein; IL-6, Interleukin-6; TNF- $\alpha$, tumor necrosis factor $\alpha$; MMP-3, matrix metalloproteinase-3; SOD, superoxide dismutase; MDA, malondialdehyde; SE, standard error; Cl, confidence interval.

can establish a link between neurodegenerative diseases and oxidative stress. However, relevant research is still lacking, especially to assess whether elderly frail patients or patients with CSVD can effectively improve disease progression and slow cognitive decline by increasing the intake of antioxidants or anti-inflammatory agents. It can be inferred from this study that reducing inflammation and oxidative stress may delay the progression of patients with CSVD, but further clinical trials and in-depth studies are needed for drug treatments to inhibit inflammation and oxidative stress. In addition, based on the current lack of understanding of cognitive weakness, the treatment of elderly patients with neurodegenerative diseases still faces considerable challenges to overcome (52).

The sample size of this study is limited, which can be expanded in future studies. At the same time, this study is a cross-sectional study, without tracking the health status of patients, especially the adverse outcomes, such as death. In future research, multidisciplinary team cooperation will be adopted to provide personalized guidance for patients with small cerebral

\section{REFERENCES}

1. de la Cruz-Cosme C, Dawid-Milner MS, Ojeda-Burgos G, GallardoTur A, Segura T. Doppler resistivity and cerebral small vessel disease: hemodynamic structural correlation and usefulness for the etiological classification of acute ischemic stroke. J Stroke Cerebrovasc Dis. (2018) 27:3425-35. doi: 10.1016/j.jstrokecerebrovasdis.2018.08.001

2. Wei W, Chen Y, Lei D, Zhang Y, Weng X, Zhou Y, et al. Plasma brain natriuretic peptide is a biomarker for screening ischemic cerebral small vessel disease in patients with hypertension. Medicine. (2018) 97:e12088. doi: 10.1097/MD.0000000000012088

3. Yang S, Yuan J, Qin W, Yang L, Fan H, Li Y, et al. Twenty-four-hour ambulatory blood pressure variability is associated with total magnetic resonance imaging burden of cerebral small-vessel disease. Clin Interv Aging. (2018) 13:1419-27. doi: 10.2147/CIA.S171261

4. Clegg A, Young J, Iliffe S, Rikkert MO, Rockwood K. Frailty in elderly people. Lancet. (2013) 381:752-62. doi: 10.1016/S0140-6736(12)62167-9

5. Dent E, Martin FC, Bergman H, Woo J, Romero-Ortuno R, Walston JD. Management of frailty: opportunities, challenges, and future directions. Lancet. (2019) 394:1376-86. doi: 10.1016/S0140-6736(19)31785-4 vascular disease and cognitive weakness and a multidisciplinary combination of frailty prevention and management.

\section{CONCLUSION}

In summary, our research results showed that the increase in serum CRP, TNF- $\alpha$, MMP-3, and MDA levels are associated with an increased risk of cognitive frailty in patients with CSVD. At present, there is no unified treatment method for CSVD. At the same time, the treatment of frailty and cognitive frailty is not clear. Studies have shown that anti-inflammatory treatment may be helpful. The clinical significance of this study is to explore the relationship between patients with CSVD with combined frailty, cognitive frailty, and inflammatory factors, and oxidative stress factors, to provide new ideas for improving the cognitive frailty of patients with CSVD.

\section{DATA AVAILABILITY STATEMENT}

The original contributions presented in the study are included in the article/supplementary material, further inquiries can be directed to the corresponding author/s.

\section{ETHICS STATEMENT}

The studies involving human participants were reviewed and approved by the Ethics Committee of the Tianjin Huanhu Hospital (No. 2019-29). The patients/participants provided their written informed consent to participate in this study.

\section{AUTHOR CONTRIBUTIONS}

JW contributed to the experiment design, manuscript draft, and data analysis. LM contributed to the experiment implementation and manuscript draft. LJ, JC, MX, WW, and PL analyzed the data. All authors read and approved the final manuscript.

6. Cunha AIL, Veronese N, de Melo Borges S, Ricci NA. Frailty as a predictor of adverse outcomes in hospitalized older adults: a systematic review and metaanalysis. Ageing Res Rev. (2019) 56:100960. doi: 10.1016/j.arr.2019.100960

7. Ruan Q, Yu Z, Chen M, Bao Z, Li J, He W. Cognitive frailty, a novel target for the prevention of elderly dependency. Ageing Res Rev. (2015) 20:1-10. doi: 10.1016/j.arr.2014.12.004

8. McCauley ME, Baloh RH. Inflammation in ALS/FTD pathogenesis. Acta Neuropathol. (2019) 137:715-30. doi: 10.1007/s00401-018-1933-9

9. Shekhar S, Cunningham MW, Pabbidi MR, Wang S, Booz GW, Fan F. Targeting vascular inflammation in ischemic stroke: recent developments on novel immunomodulatory approaches. Eur J Pharmacol. (2018) 833:53144. doi: 10.1016/j.ejphar.2018.06.028

10. Anrather J, Iadecola C. Inflammation and stroke: an overview. Neurotherapeutics. (2016) 13:661-70. doi: 10.1007/s13311-016-0483-x

11. Low A, Mak E, Rowe JB, Markus HS, O’Brien JT. Inflammation and cerebral small vessel disease: a systematic review. Ageing Res Rev. (2019) 53:100916. doi: 10.1016/j.arr.2019.100916

12. Li T, Huang Y, Cai W, Chen X, Men X, Lu T, et al. Age-related cerebral small vessel disease and inflammaging. Cell Death Dis. (2020) 11:932. doi: 10.1038/s41419-020-03137-x 
13. Wu IC, Lin CC, Hsiung CA. Emerging roles of frailty and inflammaging in risk assessment of age-related chronic diseases in older adults: the intersection between aging biology and personalized medicine. Biomedicine. (2015) 5:1. doi: 10.7603/s40681-015-0001-1

14. Hilal S, Ikram MA, Verbeek MM, Franco OH, Stoops E, Vanderstichele $\mathrm{H}$, et al. C-Reactive protein, plasma amyloid-beta levels, and their interaction with magnetic resonance imaging markers. Stroke. (2018) 49:2692-8. doi: 10.1161/STROKEAHA.118.022317

15. Rouhl RP, Damoiseaux JG, Lodder J, Theunissen RO, Knottnerus IL, Staals J, et al. Vascular inflammation in cerebral small vessel disease. Neurobiol Aging. (2012) 33:1800-6. doi: 10.1016/j.neurobiolaging.2011.04.008

16. de Oliveira Ulbrecht MO, Goncalves DA, Zanoni LZG, do Nascimento VA. Association between selenium and malondialdehyde as an efficient biomarker of oxidative stress in infantile cardiac surgery. Biol Trace Elem Res. (2019) 187:74-9. doi: 10.1007/s12011-018-1378-y

17. Khoubnasabjafari M, Soleymani J, Jouyban A. Avoid using spectrophotometric determination of malondialdehyde as a biomarker of oxidative stress. Biomark Med. (2018) 12:551-4. doi: 10.2217/bmm-2017-0437

18. Ceci R, Duranti G, Di Filippo ES, Bondi D, Verratti V, Doria C, et al. Endurance training improves plasma superoxide dismutase activity in healthy elderly. Mech Ageing Dev. (2020) 185:111190. doi: 10.1016/j.mad.2019.111190

19. Morley JE, Malmstrom TK, Miller DK. A simple frailty questionnaire (FRAIL) predicts outcomes in middle aged African Americans. J Nutr Health Aging. (2012) 16:601-608. doi: 10.1007/s12603-012-0084-2

20. Susanto M, Hubbard RE, Gardiner PA. Validity and responsiveness of the FRAIL scale in middle-aged women. J Am Med Dir Assoc. (2018) 19:659. doi: 10.1016/j.jamda.2017.08.003

21. Fried LP, Tangen CM, Walston J, Newman AB, Hirsch C, Gottdiener J, et al. Frailty in older adults: evidence for a phenotype. J Gerontol A Biol Sci Med Sci. (2001) 56:M146-56. doi: 10.1093/gerona/56.3.M146

22. Freitas S, Batista S, Afonso AC, Simões MR, de Sousa L, Cunha L, et al. The Montreal Cognitive Assessment (MoCA) as a screening test for cognitive dysfunction in multiple sclerosis. Appl Neuropsychol Adult. (2018) 25:5770. doi: 10.1080/23279095.2016.1243108

23. Larner AJ. Mini-Mental State Examination: diagnostic test accuracy study in primary care referrals. Neurodegener Dis Manag. (2018) 8:3015. doi: $10.2217 / \mathrm{nmt}-2018-0018$

24. Williams JB. Standardizing the Hamilton Depression Rating Scale: past, present, and future. Eur Arch Psychiatry Clin Neurosci. (2001) 251(Suppl. 2):II6-12. doi: 10.1007/BF03035120

25. Thompson E. Hamilton Rating Scale for Anxiety (HAM-A). Occup Med (Lond). (2015) 65:601. doi: 10.1093/occmed/kqv054

26. Kang J, Jeong YJ, Jang JH, Lee M. Risk factors for frailty in critical care survivors: a secondary analysis. Intensive Crit Care Nurs. (2021) 64:102981. doi: 10.1016/j.iccn.2020.102981

27. Kim E, Sok SR, Won CW. Factors affecting frailty among communitydwelling older adults: a multi-group path analysis according to nutritional status. Int J Nurs Stud. (2021) 115:103850. doi: 10.1016/j.ijnurstu.2020. 103850

28. Solfrizzi V, Scafato E, Lozupone M, Seripa D, Giannini M, Sardone $\mathrm{R}$, et al. Additive role of a potentially reversible cognitive frailty model and inflammatory state on the risk of disability: the Italian longitudinal study on aging. Am J Geriatr Psychiatry. (2017) 25:123648. doi: 10.1016/j.jagp.2017.05.018

29. Tay L, Lim WS, Chan M, Ye RJ, Chong MS. The independent role of inflammation in physical frailty among older adults with mild cognitive impairment and mild-to-moderate Alzheimer's disease. J Nutr Health Aging. (2016) 20:288-99. doi: 10.1007/s12603-015-0 617-6

30. Mekli K, Nazroo JY, Marshall AD, Kumari M, Pendleton N. Proinflammatory genotype is associated with the frailty phenotype in the English Longitudinal Study of Ageing. Aging Clin Exp Res. (2016) 28:413-21. doi: 10.1007/s40520-015-0419-z

31. Hsu B, Hirani V, Cumming RG, Naganathan V, Blyth FM, Wright FC, et al. Cross-sectional and longitudinal relationships between inflammatory biomarkers and frailty in community-dwelling older men: the concord health and ageing in men project. J Gerontol A Biol Sci Med Sci. (2019) 74:83541. doi: 10.1093 /gerona/glx142
32. Ma L, Sha G, Zhang Y, Li Y. Elevated serum IL-6 and adiponectin levels are associated with frailty and physical function in Chinese older adults. Clin Interv Aging. (2018) 13:2013-20. doi: 10.2147/CIA.S180934

33. Schaap LA, Pluijm SM, Deeg DJ, Harris TB, Kritchevsky SB, Newman AB, et al. Higher inflammatory marker levels in older persons: associations with 5year change in muscle mass and muscle strength. J Gerontol A Biol Sci Med Sci. (2009) 64:1183-9. doi: 10.1093/gerona/glp097

34. Inglés M, Gambini J, Carnicero JA, García-García FJ, Rodríguez-Mañas L, Olaso-González G, et al. Oxidative stress is related to frailty, not to age or sex, in a geriatric population: lipid and protein oxidation as biomarkers of frailty. J Am Geriatr Soc. (2014) 62:1324-8. doi: 10.1111/jgs.12876

35. Lei D, Sha Y, Wen S, Xie S, Liu L, Han C. Dexmedetomidine may reduce IL-6 level and the risk of postoperative cognitive dysfunction in patients after surgery: a meta-analysis. Dose Response. (2020) 18:1559325820902345. doi: 10.1177/1559325820902345

36. Chen N, Chen X, Xie J, Wu C, Qian J. Dexmedetomidine protects aged rats from postoperative cognitive dysfunction by alleviating hippocampal inflammation. Mol Med Rep. (2019) 20:2119-26. doi: 10.3892/mmr.2019.10438

37. Stomrud E, Bjorkqvist M, Janciauskiene S, Minthon L, Hansson O. Alterations of matrix metalloproteinases in the healthy elderly with increased risk of prodromal Alzheimer's disease. Alzheimers Res Ther. (2010) 2:20. doi: 10.1186/alzrt44

38. Wu C, Gao B, Gui Y. Malondialdehyde on postoperative day 1 predicts postoperative cognitive dysfunction in elderly patients after hip fracture surgery. Biosci Rep. (2019) 39:BSR20190166. doi: 10.1042/BSR20190166

39. Yao ZH, Yao XL, Zhang Y, Zhang SF, Hu JC. Luteolin could improve cognitive dysfunction by inhibiting neuroinflammation. Neurochem Res. (2018) 43:80620. doi: 10.1007/s11064-018-2482-2

40. Huang X, Hussain B, Chang J. Peripheral inflammation and blood-brain barrier disruption: effects and mechanisms. CNS Neurosci Ther. (2021) 27:3647. doi: $10.1111 / \mathrm{cns} .13569$

41. Chupel MU, Minuzzi LG, Furtado G, Santos ML, Hogervorst E, Filaire E, et al. Exercise and taurine in inflammation, cognition, and peripheral markers of blood-brain barrier integrity in older women. Appl Physiol Nutr Metab. (2018) 43:733-41. doi: 10.1139/apnm-2017-0775

42. Park JC, Han SH, Mook-Jung I. Peripheral inflammatory biomarkers in Alzheimer's disease: a brief review. BMB Rep. (2020) 53:10-9. doi: 10.5483/BMBRep.2020.53.1.309

43. Ng A, Tam WW, Zhang MW, Ho CS, Husain SF, McIntyre RS, et al. IL1beta, IL-6, TNF- alpha and CRP in elderly patients with depression or alzheimer's disease: systematic review and meta-analysis. Sci Rep. (2018) 8:12050. doi: 10.1038/s41598-018-30487-6

44. Zhou H, Lu S, Chen J, Wei N, Wang D, Lyu H, et al. The landscape of cognitive function in recovered COVID-19 patients. J Psychiatr Res. (2020) 129:98-102. doi: 10.1016/j.jpsychires.2020.06.022

45. Han $\mathrm{C}, \mathrm{Fu} \mathrm{R}$, Lei W. Beneficial effects of dexmedetomidine on early postoperative cognitive dysfunction in pediatric patients with tonsillectomy. Exp Ther Med. (2018) 16:420-6. doi: 10.3892/etm.2018.6180

46. Iadecola C, Duering M, Hachinski V, Joutel A, Pendlebury ST, Schneider JA, et al. Vascular cognitive impairment and dementia: JACC scientific expert panel. J Am Coll Cardiol. (2019) 73:3326-44. doi: 10.1016/j.jacc.2019.04.034

47. Ruan Q, D’Onofrio G, Sancarlo D, Greco A, Lozupone M, Seripa D, et al. Emerging biomarkers and screening for cognitive frailty. Aging Clin Exp Res. (2017) 29:1075-86. doi: 10.1007/s40520-017-0741-8

48. Mone P, Gambardella J, Pansini A, de Donato A, Martinelli G, Boccalone E, et al. Cognitive impairment in frail hypertensive elderly patients: role of hyperglycemia. Cells. (2021) 10:2115. doi: 10.3390/cells10082115

49. Uchmanowicz I. Oxidative stress, frailty and cardiovascular diseases: current evidence. Adv Exp Med Biol. (2020) 1216:6577. doi: 10.1007/978-3-030-33330-0_8

50. Mulero J, Zafrilla P, Martinez-Cacha A. Oxidative stress, frailty and cognitive decline. J Nutr Health Aging. (2011) 15:75660. doi: 10.1007/s12603-011-0130-5

51. Cosarderelioglu C, Nidadavolu LS, George CJ, Oh ES, Bennett DA, Walston JD, et al. Brain renin-angiotensin system at the intersect of physical and cognitive frailty. Front Neurosci. (2020) 14:586314. doi: $10.3389 /$ fnins.2020.586314 
52. Mantovani E, Zucchella C, Schena F, Romanelli MG, Venturelli M, Tamburin S. Towards a redefinition of cognitive frailty. J Alzheimers Dis. (2020) 76:83143. doi: 10.3233/JAD-200137

Conflict of Interest: The authors declare that the research was conducted in the absence of any commercial or financial relationships that could be construed as a potential conflict of interest.

Publisher's Note: All claims expressed in this article are solely those of the authors and do not necessarily represent those of their affiliated organizations, or those of the publisher, the editors and the reviewers. Any product that may be evaluated in this article, or claim that may be made by its manufacturer, is not guaranteed or endorsed by the publisher.

Copyright $\odot 2022 \mathrm{Mu}$, Jiang, Chen, Xiao, Wang, Liu and Wu. This is an open-access article distributed under the terms of the Creative Commons Attribution License (CC $B Y)$. The use, distribution or reproduction in other forums is permitted, provided the original author(s) and the copyright owner(s) are credited and that the original publication in this journal is cited, in accordance with accepted academic practice. No use, distribution or reproduction is permitted which does not comply with these terms. 